\title{
Challenges and Problems Affecting the Development Emergency Medical Services in Kosovo
}

\author{
Basri Lenjani ${ }^{1 *}$, Merima Šš̌ić ${ }^{2}$, Verica Mišanović ${ }^{2}$, Kenan Ljuhar ${ }^{3}$, Dardan Lenjani ${ }^{1}$
}

Received: 06 June 2021 / Accepted: 01 July 2021 / Published online: 20 July 2021

This article is published with open access at https://journal.astes.org.al

(C) The author(s) 2021. \& Copyright (C) 2021, the Albanian Society for Trauma and Emergency Surgery

(c) The Albanian Journal of Trauma and Emergency Surgery is an Open Access Journal. All articles are distributed under the terms of the Creative Commons Attribution Non-Commercial License: http://creativecommons.org/licenses/by-nc/4.0/) which permits unrestricted non-commercial use, distribution, and reproduction in any medium provided the original work is properly cited.

\begin{abstract}
Emergency medical service is organized as a separate field of health activities in order to provide uninterrupted emergency medical care for citizens who due to illness or injury have directly threatened the life, certain organs or certain parts of the body respectively cut the optimal time of occurrence of the emergency until the start of the final treatment process. Emergence clinic for 2020. Year ED over 100.000-cases. The emergency health system doesn't have a consolidated network and integrated emergency medical services. Emergency health services in Europe are being challenged by changes in life dynamics, scientific advancements, which do increase the request to further improve the way of delivering emergency services. Health-system resilience can be defined as the capacity of health actors, institutions, and populations to prepare for and effectively respond to crises, to maintain core functions when a crisis hits, and - informed by lessons learned during the crisis to reorganize if conditions require it. Emergency clinic today at UCCK offers an area of $507 \mathrm{~m}^{2}$, with 22 beds in the living room (1 bed per 100,000 population). Compliance with the law on emergency medical care, support, and improvement of EMS creating a special budget for EMS. EMS Independence (Decentralization). Budget, Management, accreditation, initiation of a project of systematization doctors of nurses in an integrated system. Regulation of administrative and legal infrastructure for EMS. The increase in salary (during holidays, weekends), shortening working hours for EMS, beneficial path (stress, risk, complexity, infections, first contact with the patient), the extension of annual leave. Functionalization of the Permanent National Center for Education EMS training, licensing, relicensing (medical staff) Quality control or EMS quality.
\end{abstract}

Keywords: Emergency clinic, patient, organized, challenged emergency medical, system referral, EMS

\section{Introduction}

EMS system includes four basic steps: Give care until help takes over. Recognize that an Emergency Exists: Keep people from responding, Good Samaritan Laws. When you should stop giving care: During these assessments, statewide EMS systems are evaluated on the basis of 10 essential

Original article, no submission or publication in advance or in parallel

* Corresponding author:

Prof.Asoc. Basri Lenjani MD.MSc.PhD

$\bowtie$ basrilenjani@yahoo.com

1 Emergency Clinic, University Clinical Centre of Kosovo.

2 Emergency Center, General Hospital "Prim. Dr. Abdulah Nakaš" Sarajevo. BOSNIA and HERZEGOVINA

3 Emergency Medical Center of Canton Sarajevo, 71000 Sarajevo, BOSNIA and HERZEGOVINA components: regulation and policy, resource management, human resources and training, transportation, facilities, communications, public information and education, medical direction, trauma systems, and evaluation.

The University Department of Medical Emergency is a referral institution of medical emergency in Kosovo located in University Clinical Center of Kosovo and represents the synthesis of a: a) Administrative; b) Academic and c) Clinical complex. We would like to thank medical staff of Emergency Clinic of the University Clinical Center of Kosovo. Collaborations. Emergency medical service is organized as a separate field of health activities in order to provide uninterrupted emergency medical care for citizens who due to illness or injury were directly threatened the life, certain organ or certain parts of the body, respectively cuts optimal time of occurrence of the emergency until the start of the final treatment process. Emergency departments (EDs) play an important public health role during emergencies and on a regular basis by providing access to emergency care to all patients regardless of their ability to pay. 


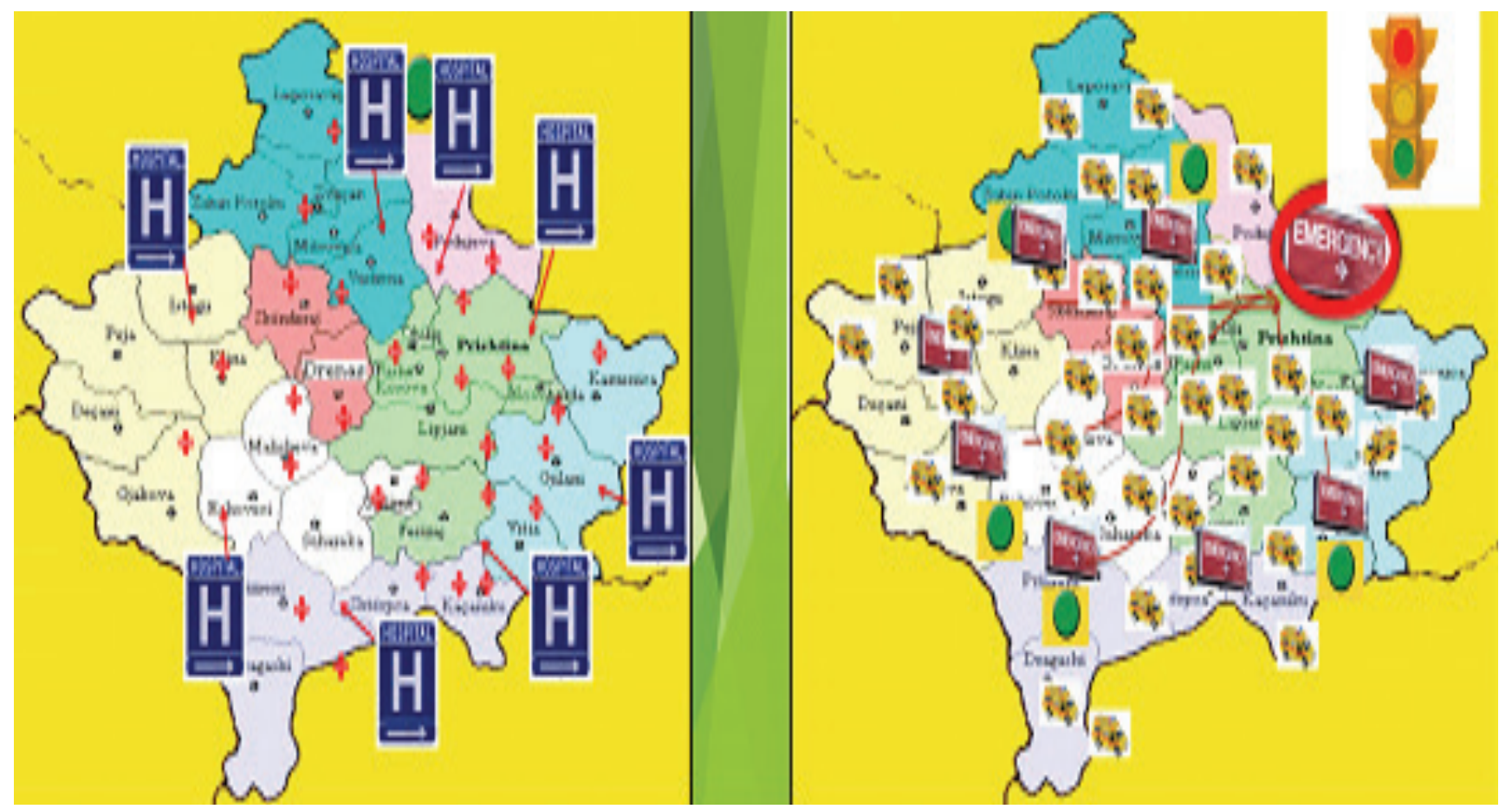

Figure 1. Kosovo with 8 regional hospitals, 8 emergency centers and a UCK emergency clinical reference nationwide

Mission. Providing emergency medical care within the golden hour and increasing the chances of survival in regular and emergency conditions. Our mission is to provide high quality and timely care to patients requiring emergency treatment; to offer outstanding educational opportunities for medical, nursing and allied health personnel, and to further medical knowledge through the pursuit of research. We strive to develop an environment that stresses compassion and respect for our patients and each other; that employs the highest ethical standards; that utilises resources in an efficient manner; and that promotes public health.

Vision. Improving services within the golden hour and increasing the chances of survival in regular and extraordinary conditions.

Improving emergency healthcare and research in Kosovo is a goal, which cannot be achieved in isolation. Over the years we have been successful in establishing crucial linkages with academic and health care institutes (local and international), NGOs, government bodies and international organizations such as the WHO. We believe multiple simultaneous steps are necessary to solve ED crowding. We present 10 putative solutions with commentary on actions at our institution to counter the problem. Hospital-Based Emergency Care addresses the difficulty of balancing the roles of hospital-based emergency and trauma care, not simply urgent and lifesaving care, but also safety net care for uninsured patients, public health surveillance, disaster preparation, and adjunct care in the face of increasing patient volume and limited resources

Pressure for change. Emergency health system doesn't have a consolidated network and integrated emergency medical services. In economic terms, investments in emergency medical services do not really generate financial benefits, but may save human lives. However, is considered as very important, affecting the quality of life as well as implicating further economical costs for a scoiety, rehabilitation of a patient who underwent a life threatening situation. Emergency health system does'nt have a consolidated network and integrated emergency medical services. Considering the demographic, economic and social changes in Kosovo, the goal is empowering the patient's expectation and as well as respond to a better level of emergency medical careIn economic terms, investments in emergency medical services do not really generate financial benefits, but may save human lives.

However, is considered as very important, affecting the quality of life as well as implicating further economic costs for a scoiety, rehabilitation of a patient who underwent a lifethreatening situation. Emergency health services in Europe are being challenged by changes in life dynamics, scientific advancments, which do increase the request to further improve the way of delivering the emergency services. Furthermore, it can be given the directions for developing emergency care levels and intersectional cooperation, too. This fact indicates that the referral system of emergency services fail and contributes to ineffective usage of available resources.

Insufficient spatial capacity. Health-system resilience can be defined as the capacity of health actors, institutions, and populations to prepare for and effectively respond to crises, to maintain core functions when a crisis hits, andinformed by lessons learned during the crisis - to reorganize if conditions require it. Nowday, available emergency services in UCCK are given in a space of 507m2, 22 daily- 
stay beds ( 1 bed per 100,000 inhabitants). Emergence clinic for 2020. year EMS over 100. 000 cases.

Confrontations challenge. Need for further professional training, especially focused to emergency physicians and their assistants. Lack of standardization (clinical service and definitions). Poor data retentions of the patient (medical cards and records). Lack of diagnostic capacities to perform basic diagnostic tests and lifesaving procedures. Lack of a proper referral system. Changing of the system. In some cases, also contributes to the circumvention of this institution and emergency assistance are being requested at other institutions. Lack of laboratory and radiological diagnostic services in Emergency Department fairly represent a difficulty in diagnosis and treatment. This is mainly due to lack of equipment, regular supply of the drugs, consumable materials and medical staff Presents a problem in itself and lack of protocols that will enable decision making emergency, correct, as in diagnosis, treatment based on algorithms, techniques of selection according to international standards.

Problems of emergency department. Five challenges facing emergency medicine; Capacity. The greatest challenge is and has always been emergency department capacity. Length of stay. Hospitals have consistently looked at ways to improve emergency department traffic flow, declining reimbursements; declining reimbursement poses many issues, staffing, technology

\section{Objectives}

Providing quality health care services. Strengthening the monitoring role of $\mathrm{MH}$. Sustainable financing of emergency services. Providing human and capital resources.

Objectives and implementation measures. Providing the safe emergency services. Offering integrated emergency
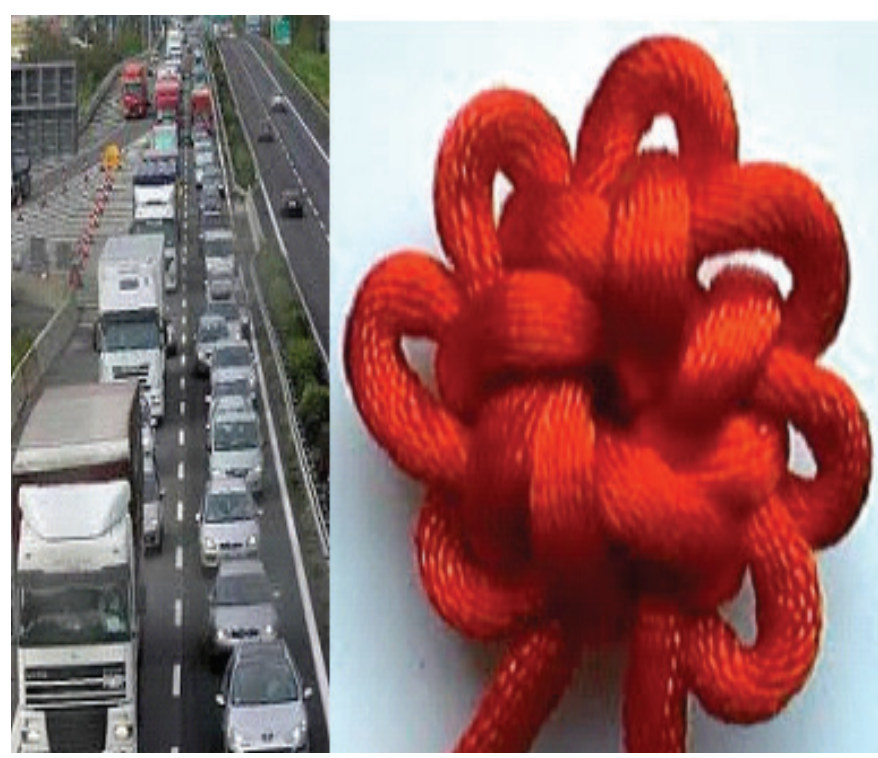

services. Ensuring the minimum professional framework for the operation of the Emergency Center. Advancing scientific research

Policy and Planning. $\mathrm{MH}$ still fits with constant changes and developments in society, through policies and planning for the future. The spectrum of emergency services in UCCK is influenced by the adoption of new medical technologies and unbalanced supply of manpower in emergency. Information and communication technologies (ICT) applied to emergency health care have a significant impact for the functionalisation of health services which are currently missing. This makes difficulties on cooperation and coordination between the health care levels and other sectors (eg. health and social sector, primary, secondary, tertiary helath care levels ect).

The process of developing the Emergency Services in UCCK is based on the format offered by WHO and tend in the context of health care, to define a longterm conturs of strategic and development plan of the Government for adapting and operating it with a socio-economic background of Kosovo. A good document also defines the structures, activities and time limits to contribute the implementation of this plan.

Development process in Kosovo Emergency Services. A first step is to identify reasons for reforming the Medical Emergency Services. The second step is to achieve the objectives and goals, as well as implementation of emergency medical services. However, new challenges that are set by very young population, the dramatic increase in emergency situations, increasing the number of accidents, threats by epidemics and pandemics, etc, made neccesary the emergency health care services to be reviewed.

Strengthening the monitoring role of $\mathrm{MH}$. Ensuring the implementation of standards and norms defined in this document and definition of those that are missing.

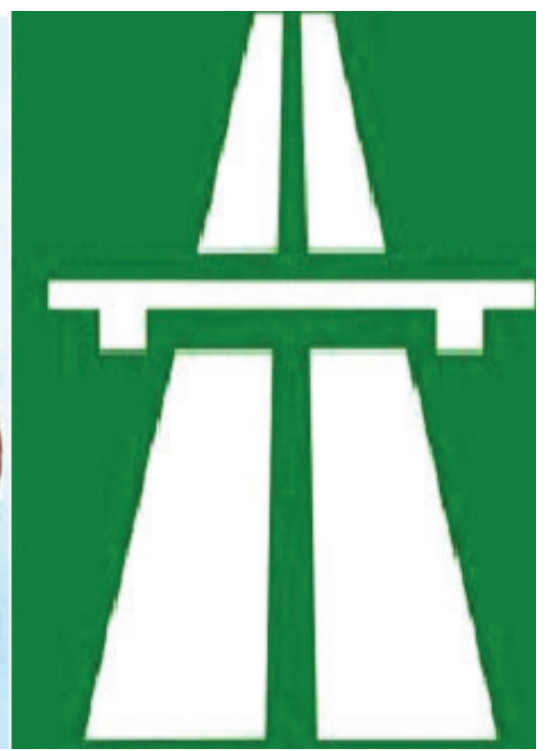

Foto. 1. Chaotic referral system, current situation. 
Ensuring the continued implementation of the licensing process. Initiating of the process for medical emergency services accreditation. Regular surveillance of the quality of emergency services. Supervision of management process. Functionality of a health information system. Defining the basic package cost for medical emergency services. Providing the necessary finacial support for medical emergency services

Providing human and capital resources. Provideing the educational and CME programs for specialist of Emergency Medicine and other professional staff in Emergency Centre. Motivation of staff working in the Emergency Center. Providing sufficient amount of medicines and consumables. Establishment of the mechanism for evaluation and promotion of medical and communication technology

Are there solutions. Compliance with the law on emergency medical care. Support and improvement of Emergency medical services.

Creating a special budget EMS. Redesigning the Delivery of Care Through Emergency Department Quality Improvement As the crisis of emergency care grows, hospitals have more pressure to make improvements in addition to the many other pressures facing the healthcare industry. Redesigning the delivery of emergency care involves a four-step approach. Begin with the bigger aim and ask "What do we need to best deliver emergency care?" Improving emergency care ties into the Triple Aim of healthcare improvement: improving the patient experience of care, improving the health of populations, and reducing the per capita cost of healthcare. Create a vision and support for a data-driven systems approach to improving emergency care.

Many times, a department will embark on an improvement project, but don't think about how it will affect the flow of other teams or departments. Instead, it can be helpful to create an ED Joint Practice

Team where anyone who touches the patient is part of the care team. This helps improve communication between departments to avoid unforeseen consequences of changes to other departments. Several of these health system factors are in flux, and how such changes play out may improve or harm ED function.

For example, the growth of urgent care, retail clinics, and efforts to expand access to insurance and better coordinate care may improve ED operations, but these changes may have unintended consequences and may not affect all EDs equally.

Using an analytics platform, develop an ED analytics application that provides insight into performance. If an ED with 65 beds has 130 patients come through the doors on any given day, but the OR doesn't know, nor does the hospital care control center, the ED is operating in isolation without the ability to resolve issues or foresee them in advance. Using an analytics application such as ED Explorer, frontline leadership and executives have the ability to track throughput, and obtain actionable data necessary for demand/capacity management. Engage frontline staff, key stakeholders, patients, and families in improvement. Improving ED throughput is much more than an emergency department initiative. It must include the entire system of care for real, lasting change to occur. Delivery system changes are also occurring in the midst of populationlevel changes, which may increase the need for ED services because the population is aging with higher rates of chronic disease

\section{Discussion and Conculsions}

Emergency Department Quality Improvement: Transforming the Delivery of Care, This article is based on a 2018 Healthcare Analytics Summit (HAS 18) presentation given by Linda Hummel, MS, BSN, RN, Vice President of Quality and Patient Safety at Mission Health System and Rick Lee, MSN, RN, CEN, NE-BC, Executive Director of Em.More than half of all U.S. hospitals report overcrowding in the Emergency Department (ED), one-third report an increase in ambulance diversion, and 90 percent report they frequently operate at or over capacity, risking the ability to effectively provide emergency care to those who need it and contributing to patient dissatisfaction.

Overcrowded EDs cause problems for both hospital patients and staff, such as increased wait times, length of stay (LOS), medical errors, and mortality rates, as well as financial losses to hospitals. A data-driven systems approach can dramatically improve every aspect of emergency care, improving wait times and throughput, reducing left without being seen rates, and improving patient satisfaction. Common Problems with Emergency Care. Crowding in the ED has become a widespread problem in hospitals across the country for two primary reasons: emergency medicine is the only specialty with a federal mandate to provide care to patients seeking treatment, and a shortage of primary care providers has forced more sick people to seek treatment in hospital emergency rooms. This overcrowding leads to a number of common problems with emergency care that can be seen as markers of hospital health.

The emergency clinic faced a large number of problems and challenges such as space capacity, medical equipment, costly drugs, ED overcrowding, referral system, education and training in its ability to provide effective emergency medical services. large proportion of patients were coming out of visiting ED and, on average, patients had immediate access to see a qualified emergency medical provider. Where within 24 / h, 320 cases were required or every 4.4 minutes they arrive from one patient and perimismii improving the quality of emergency health services in ED was difficult to raise this component and improve patient flow, patient experience for services with medical in ED with a datadriven approach. But the key problem is the referral system as a component not regulated at the country level. In just over a year, they dramatically improved all aspects of the ED run, including. 
As the crisis of emergency care grows, hospitals have more pressure to make improvements in addition to the many other pressures facing the healthcare industry. Redesigning the delivery of emergency care involves a four-step approach. As according to the currently valid legal acts, the organization of EMS activities does not meet the requirements of modern emergency medicine, it is necessary to change the legal ones at the levels that regulate and regulate this activity. It is necessary to harmonize them with modern European laws related to this area. This is the first step, a precondition for changes in the organization in general.The proposed priorities for the development of emergency medical care are; The proposed priorities for the development of emergency medical care are; Financing, organization and education. Cooperation with: Faculty of Medicine and. Ministry of Education. Mandatory legal protection for emergency medical personnel at three levels of EMS; Primarily, Secondary and Tertiary.

EMS Independence (Decentralization)L; Budget, Management, Accreditation, Initiation of a project of systematization of nurses in an integrated system. Without solutions and without government support there will be no progress. A number of health system factors affect ED care, including insurance coverage; the availability of inpatient hospital care; and the availability of outpatient providers, in general, after hours, and their willingness to accept particular insurance types

Improving how EDs function will require system-wide changes in health care delivery, as ED care is affected by a number of factors in the health care delivery system beyond an ED's control.

\section{Recommendation}

Compliance with the law on emergency medical care. Support and improvement of EMS. Creating a special budget for EMS. EMS Independence (Decentralization), Budget, management, accreditation, initiation of a project of systematization of nurses in an integrated system, development of protocols, algorithms and clinical guidelines primarily, secondary and tertiary regulation of administrative and legal infrastructure for EMS, the increase in salary (during holidays, weekends), shortening working hours for EMS, beneficial path (stress, risk, complexity, infections, first contact with the patient), the extension of annual leave, functionalization of the Permanent National Center for Education EMS, training, licensing, relicensing (medical staff) Quality control or EMS quality, cooperation with: faculty of Medicine and ministry of Education, mandatory legal protection for emergency medical personnel at three levels of EMS; primarily, secondary and tertiary

\section{If you want success; they do not agree with the present.} Roberto Goizueta

\author{
Abbreviations \\ ED: Emergency department \\ EMS: Emergency Medical System \\ WHO: Word Health Organization. \\ UCCK: University Clinical Center of Kosovo
}

Acknowledgement; We would like to thank medical staff of Emergency Clinic of the University Clinical Center of Kosovo.

Financial support and sponsorship: Nil.

Conflicts of interest: There are no conflicts of interest.

\section{References}

1. Official Gazette of the Republic of Kosova / no. 38 / 21 November 2016, Pristina Law no. 05/1-024 for Emergency Medical Service, 1-3.

2. Weber EJ, Mason S, Freeman JV, et al. Implications of England's four-hour target for quality of care and resource use in the emergency department. Ann Emerg Med. 2012;60(6):699-706.

3. Toh D, Thompson CH, Thomas J, et al. Emergency department overcrowding and mortality after the introduction of the 4-hour rule in Western Australia. Med J Aust. 2012;196(8);499-500.

4. Agency for Health Care Research and Quality, "Overview of Emergency Department Visits in the United States, 2011,” Statistical Brief \#174, 2014.

5. Robert W. Derlet, MD and John R. Richards, MD Ten Solutions for Emergency Department Crowding, West J Emerg Med. 2008 Jan; 9(1): 24-27.

6. Vila-Roel C, Guo X, Holroyd BR, et al. The role of full capacity protocols on mitigating overcrowding in EDs. Am J Emerg Med. 2012;30(3):412-420.

7. 6 Agency for Health Care Research and Quality, "Overview of Emergency Department Visits in the United States, 2011," Statistical Brief \#174, 2014.

8. Hospital-Based Emergency Departments: Background and Policy Considerations Elayne J. Heisler Specialist in Health Services Nancy Leigh Tyler Research Associate December 8, 2014

9. Emergency Department Design Guidelines (G15)3 of 77; The Australasian College for Emergency Medicine (ACEM).

10. Pollaris G, Sabbe M. Reverse triage: more than just another method. Eur J Emerg Med. 2015;0(0):1-8.

11. Powell ES, Khare RK, Venkatesh AK, et al. The relationship between inpatient discharge timing and emergency department boarding. J Emerg Med. 2012;42(2):186-196. 